\title{
Using a Body Scanner in Assessing Perceptions of Attractiveness: Cross-Regional Study
}

\author{
Mariné Aghekyan · Dong-Eun $\mathrm{Kim}^{\dagger} \cdot$ Margaret Lichty \\ Dept. of Family \& Consumer Sciences, California State University - Long Beach \\ Received June 12, 2013; Revised August 3, 2013; Accepted August 9, 2013
}

\begin{abstract}
Previous research reported that body size measured as Body Mass Index (BMI) and body shape measured as waist-hip-ratio (WHR) are two essential factors that form the perceptions of body attractiveness. However, the debate on the importance of BMI versus WHR is ongoing. In addition, scholarly articles, that focus on the role of geographic and cultural variability on perceptions of attractiveness, report inconsistent results. Some suggested that according to globalization and the rise of mass media, geographic variability of perceptions of attractiveness have been altered, while others believed that it remains. This study cross-regionally investigates the role of body size and body shape on the perceptions of female attractiveness. Samples were composed of 107 female college students in Alabama and 107 female college students in California. Participants viewed 27 three-dimensional body scan images of women in three body shapes (pear, hourglass, and rectangle) and three body sizes (underweight, normal weight, and overweight) and rated their perceptions of attractiveness. Images were shown to students in a random order with an overhead projector and Microsoft Office PowerPoint software. A three-way mixed-ANOVA was conducted to analyze the data. The results of the study showed that some regional differences exist between the two sample groups. However, regardless of the regional difference, hourglass shapes were perceived to be the most attractive shape and underweight sizes were perceived to be the most attractive size for both samples.
\end{abstract}

Key words: Cross-regional, Body attractiveness, Body shape, Body size, 3D body scanning

\section{Introduction}

The subject of physical beauty has been an important topic for artists, writers, philosophers, and researchers throughout the ages in various cultures. The publicly desirable body shape of the attractive woman has been changing for centuries. Although modern society has obsession with unrealistic thin bodies, pear shape and rectangular shape were once ideal body types in the past (Berg, 2000). Additionally, the disputes regarding the influence of geography, culture, and ethnicity on perceptions of body attractiveness still exists or has been altered due to the rise of globalization

${ }^{\dagger}$ Corresponding author

E-mail: dong-eun.kim@csulb.edu have not been settled. Therefore research on the body characteristics people perceive as attractive and the influence of geographical variability on people's perception of attractiveness in today's society are worthy of further investigation.

The research studies examining perceptions of body attractiveness consistently have taken two different directions. One school based its studies on the evolutionary psychology theories of mate selection process and found that body shape measured as the waist-hipratio (WHR) was the major cue of assessing body attractiveness (Singh, 2006; Singh \& Singh, 2011). Another groups of researchers reported that body size measured as Body Mass Index (BMI) was a stronger cue in assessing perceptions of body attractiveness (Kościński, 2013; Smith et al., 2007a, 2007b; Tovée

(C) 2013, The Korean Society of Clothing and Textiles. All rights reserved. 
et al., 2002; Wilson et al., 2005) These studies reported significant relationships in perceptions of body attractiveness and body size, and also found that body size was a stronger cue used to assess perceptions of body attractiveness rather than body shape (Aghekyan et al., 2012; Kościńnki, 2013; Swami \& Tovée, 2005, 2007). Additionally, some of these researchers have extended the topic of female attractiveness into cross-cultural studies and examined whether the ideal female figure was culturally stable or had cultural variations (Brooks et al., 2010; Dixson et al. 2010a; Fallon, 1990; Mazur, 1986; Swami et al., 2010).

Thus the first aim of the present study was to assess the effect of the body size and body shape on perceptions of body attractiveness by using 3D body scan images. Three body shapes (hourglass, rectangle, and pear shape) in three body sizes (underweight, normal weight, and overweight) were used in assessing the effect of body shape and body size in attractiveness perceptions. By using realistic representation of women bodies with large variability of body shape and body size, the researchers aimed to achieve a new perspective in understanding the role of body size and body shape in perceptions of attractiveness. The second aim of the present study was to expand the topic into a cross-regional focus in assessing perceptions of body attractiveness.

The specific research questions were the following:

(1) Do body shape differences influence perceptions of attractiveness by college students in Alabama and California?

(2) Do body size differences influence perceptions of attractiveness by college students in Alabama and California?

(3) Are the perceptions of body attractiveness different between Alabama and California college students?

\section{Literature Review}

\section{Literature on Criterion of Body Attractive- ness}

There are two main directions in the academic literature on the issue of body attractiveness criterion.
Singh (1993) suggested that the amount of fat, and most importantly its distribution, determined female body attractiveness. Fat distribution was measured by WHR, which was used to classify body shapes. The most attractive women were generally rated as those with 0.7 WHR (Dixson et al., 2010b, 2010c; Henss, 1995; Singh, 1993, 1994a, 1994b). This group of researchers suggested that WHR played a critical role in the evaluation of women's body attractiveness.

Singh (1993) used 12 line drawings with WHR ranging from 0.1 to 0.7 . However, other researchers noted that Singh's findings could be affected by the small variability within WHR ratio (Tassinary \& Hansen, 1998). Tassinary and Hansen (1998) criticized the fact that the majority of studies in this area were restricted by using the set of line drawings developed by Singh (1993) as a stimulus material. Forestell et al. (2004) discussed the involvement of body weight and shape factors in ratings of female attractiveness and replicated previous research conducted by Singh (1993, 1994a, 1994b). Forestell et al. (2004) noted that body weight, size, and WHR interacted to affect the rating of body attractiveness. It was also noted that the use of silhouettes could compromise the validity of their results and using more realistic stimuli such as photographic images and 3D images was recommended for future studies.

Meanwhile, group of researchers in the Textiles and Apparel field analyzed female body shape considering the whole body and not just waist and hip ratio. Connell et al. (2006) developed Body Shape Assessment Scale $^{\mathcal{O}}\left(\mathrm{BSAS}^{\mathcal{C}}\right)$ to evaluate whole body and component body parts of female body. This scale was used to assess female body shape based on front and side views for female shape classifications. Research studies on body image, size, cathexis, and attractiveness in the field of Textiles and Apparel widely consider the whole body when defining body shape (Aghekyan et al., 2012).

The other direction promoted by Toveé and other researchers suggested that body size measured by BMI is the main determinant in perceptions of body attractiveness (Kościński, 2013; Smith et al., 2007b; Toveé et al., 1998, 2002, 2003). BMI is a number that expresses the body build of a person, which is related to several disease risks. BMI is calculated by weight (in 
kilograms) and height (in meters) ratio $\left(\mathrm{kg} / \mathrm{m}^{2}\right)$. The method used in Toveé's studies (Toveé et al., 1998, 2002, 2003) was relatively more advanced and totally different than that used in previous research. Photo images (Toveé et al., 1999) and real body images (Toveé et al., 2003) were utilized instead. The results showed that the most attractive bodies had an average BMI of 20 and their WHR did not approach 0.7 as was suggested by Henss (1995) and Singh (1993). It was concluded that participants concentrated more on body size than body shape while identifying their ideal body. Recent studies (Kościński, 2013; Smith et al., 2007b) also agreed that BMI was the primary predictor of attractiveness although WHR had a significant effect on attractiveness perception.

\section{Geographic Variability in the Context of At- tractiveness Perceptions}

The existing literature on the role of geographic and cultural variability on perceptions of attractiveness had been conducted in mid 1980s and 1990s and reported inconsistent results. Some suggested that due to the globalization and the rise of mass media, geographic variability has been altered, while others believed that it still exists. According to Mazur (1986), the geographic variability in the perception of beauty, which existed in earlier periods, has been altered due to the rise of mass media. To support this statement Hogg and Graham (1995) investigated the determinants of human beauty and suggested that the perception of beauty was not based on cultural influences. However, Fallon (1990) wrote two disparate beliefs about the determinants of body attractiveness in each culture: "biological determinism" and "culture". According to his theory, each cultural group has different beauty standards and, naturally, the perception of attractiveness varies from culture to culture. The question about factors that constitute an universal criterion of attractiveness has many assumptions. For some cultures there are standards of body attractiveness that are predictable (Furnham \& Alibhai, 1983; Furnham \& Baguma, 1994), whereas for other cultures these standards are very different (Bush et al., 2001; Furnham \& Nordling, 1998; Furnham et al., 1997; Gupta et al., 2001).
More recent studies focusing on the impact of mass media and television on body dissatisfaction also reported inconsistent results (Jung \& Lennon, 2003; Jung $\&$ Peterson, 2007). Brooks et al. (2010) reported that attractiveness rating by Hong Kong Chinese and Australian men and women showed strong cross-cultural consistency. This result was most likely because they shared cultural similarities of living in urban and developed place with shared media exposure. A study by Swami et al. (2010) conducted in 10 major world regions confirmed the similar desire for thin ideal in highsocioeconomic-status sites; however, also confirmed significant cross-regional differences in the ideal female figure and body dissatisfaction across the sites. Considering this inconsistency in previous studies and rapid rate of globalization in recent decade, questions as (1) if globalization altered all the cultural, geographical, and ethnic differences in perceptions of attractiveness; and (2) if there is a universal criterion of attractiveness - remains unanswered.

\section{Methods}

A 2 (regions: Alabama and California) $\times 3$ (body shapes: rectangle, hourglass and pear) $\times 3$ (body sizes: underweight, normal, and overweight) mixed factorial design was used. A three-way mixed- ANOVA was conducted to compare the perception of attractiveness according to the region, body shape, and body size.

\section{Sample}

For this study, samples were selected in California and Alabama. A convenience sample of 214 female college students with age of nineteen years old or older composed a sample of this study (107 in Alabama and 107 in California), with the majority of participants being under the age of 25 (Alabama: 99.1\%; California: $85 \%$ ). The selection of these states was justified by the fact that residents of these two states are very different: California is known as a state of having a very diverse population and culture with predominantly liberal social values. According to the U. S. Census Bureau, California population in 2012 is comprised of 39.4\% Caucasian, 38.2\%, Hispanic/Latino, 
13.9\% Asian, and 6.6\% African American (United States Census Bureau, n.d.). On the other hand, the population and culture in Alabama are more homogeneous and conservative; Alabama population in 2012 is comprised of $66.6 \%$ Caucasian, 26.5\% African American, 4.1\% Hispanic/Latino, and 1.2\% Asian.

\section{Stimulus and Data Collection Procedure}

In previous studies on body attractiveness, the use of more realistic stimuli was suggested for future studies to increase study validity. One substitutive tool that is becoming to be popularly used for body image studies is a 3D body scanner (Brooks et al., 2010; Price et al., 2013). Therefore, in this study, body scan images obtained from a $[\mathrm{TC}]^{2} 3 \mathrm{D}$ body scanner were used to assess perceptions of female attractiveness. The $3 \mathrm{D}$ body scanner used white light cameras to capture a person's body image and had capacity to extract hundreds of body measurements within a few seconds. The image is a realistic representation of a person. For the present study overall 27 suitable body scan images were selected to be used as stimuli (Fig. 1). The researchers reviewed and analyzed a pool of more than two hundreds of body scan data at Auburn University. The selections were made to represent three BMI levels (underweight, normal weight, and overweight) in three body shapes (hourglass, rectangle, and pear). $\mathrm{BMI}$ is an index of weight-for-height that is commonly used to screen for weight categories of people (World Health Organization, n.d.). Adults' BMI of less than 18.50 is underweight category, BMI of 18.50 to 24.99 is normal category, and BMI of higher than 25.00 is overweight category. This same rule suggested by World Health Organization (n.d.) was followed in the present study in order to classify the body scan images into three BMI categories. Body shapes of the scans used for the current study were analyzed based on shoulder, waist, and hip relationships using BSAS $^{\mathcal{C}}$ scale (Connell et al., 2006).

Within each BMI category three body shapes were classified as follows: hourglass, rectangle, and pear shape. First, in the underweight category, there were nine images representing all three shapes: 1) three underweight/hourglass shape figures with BMI of 16.8 , 16.9 , and $17.4 ; 2$ ) three underweight/pear shape figures with BMI of 16.9, 16.9, and 17.1; and 3) three underweight/rectangle shape with the BMI of 17.2, 17.2, and 17.3. Second, in the normal weight category, there were also nine images representing all three shapes: 1) three normal/hourglass shape figures with BMI of 22.54, 21.52, and $23.65 ; 2$ ) three normal/pear shape with BMI of 21.52, 20.49, and 23.45; and 3) three normal/rectangle shape with BMI of 20.42, 22.36, and

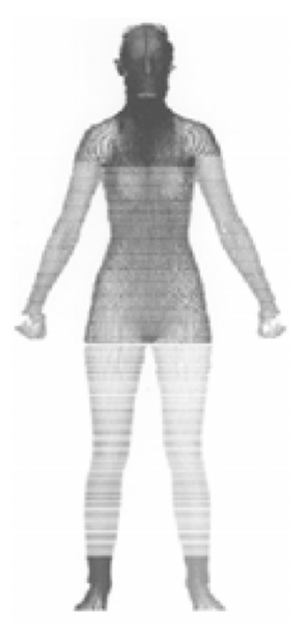

Underweight

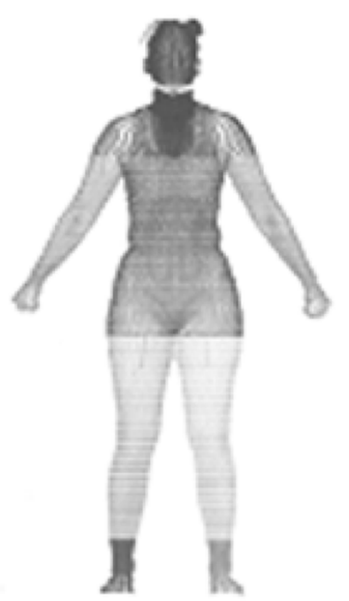

Normal weight

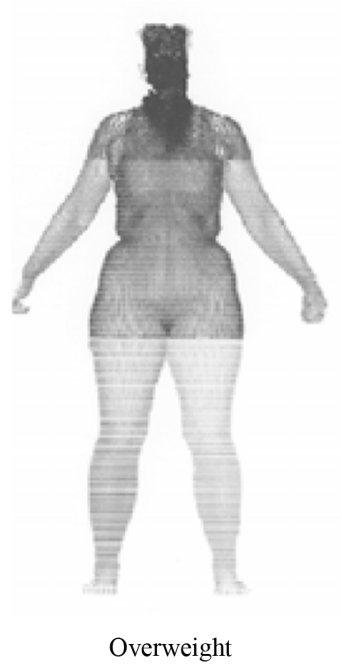

Overweight

Fig. 1. An example of body scan images of women with a pear shape. 
21.7. Third, in the overweight category, there were also nine images representing all three shapes: 1) three overweight/hourglass shape figures with BMI of 26.78 , 26.28 , and 28.05 ; 2) three overweight/pear shape figures with BMI of 27.1, 26.16, and 29.22; and 3) three overweight/rectangle shape figures with BMI of 25.98 , 25.79, and 29.33.

Participants in both regions randomly viewed the stimulus with 27 three-dimensional body scan images of women in three body shapes and three body sizes on Microsoft Office PowerPoint slides. Participants viewed each body scan separately in a random order. In other words, each scan showed a body image in any BMI level and body shape. Participants were asked to rate the attractiveness of each body scan image on a five-point Likert scale $(1=$ Very Unattractive to $5=$ Very Attractive).

\section{Data Analysis}

The data were analyzed using the SPSS 19 statistical package. The statistical analyses conducted for this research were descriptive analysis, 3-way mixed-
ANOVA, and Duncan's multiple range test.

\section{Results and Discussion}

\section{Participant Demographics}

Descriptive statistics were conducted to analyze participants' demographic characteristics. The two sample groups were different in ethnic background; the majority of the participants in Alabama were Caucasian $(92 \%, n=98)$ with a small number of African American $(5.6 \%, \mathrm{n}=6)$ and Hispanic $(0.9 \%, \mathrm{n}=1)$. Participants in California showed more diversity with Caucasian $(46.7 \%, \mathrm{n}=50)$, Asian $(21.5 \%, \mathrm{n}=23)$, Hispanic $(20.6 \%, \mathrm{n}=22)$, and African American (6.5\%, $\mathrm{n}=7)$. The results confirmed that California is a state of very diverse population and Alabama is more homogeneous. The majority of participants in both sample groups had normal BMI (Alabama: $81 \%, \mathrm{n}=81$; California: $77.6 \%, n=83)$. The Alabama sample group had slightly more participants in underweight range (Alabama: $20.6 \%, \mathrm{n}=22$; California: $8.4 \%, \mathrm{n}=9$ ) and slightly fewer participants in overweight range (Alaba-

Table 1. Three-way mixed-ANOVA result: perception of attractiveness according to region, body shape, and body size

\begin{tabular}{|c|c|c|c|c|}
\hline \multicolumn{2}{|c|}{ Source of Variance } & $\frac{d f}{d f}$ & $\overline{F F}$ & 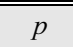 \\
\hline \multirow{3}{*}{ Main Effect } & Region & 1 & $13.92 * * *$ & $<.001$ \\
\hline & Body Shape & 2 & $72.84 * * *$ & $<.001$ \\
\hline & Body Size & 2 & $1114.02 * * *$ & $<.001$ \\
\hline \multirow{3}{*}{ 2-way Interaction Effect } & Region $\times$ Body Shape & 2 & $3.09 *$ & 0.05 \\
\hline & Region $\times$ Body Size & 2 & 0.46 & 0.63 \\
\hline & Body Size $\times$ Body Shape & 4 & $90.28 * * *$ & $<.001$ \\
\hline \multirow[t]{2}{*}{ 3-way Interaction Effect } & Region $\times$ Body Size $\times$ Body Shape & 4 & 1.78 & 0.13 \\
\hline & & & $M$ & $S D$ \\
\hline \multirow{2}{*}{ Region } & AL & & 2.62 & 0.36 \\
\hline & $\mathrm{CA}$ & & 2.80 & 0.37 \\
\hline \multirow{3}{*}{ Body Shape } & Rectangle & & $2.62^{\mathrm{b}}$ & 0.42 \\
\hline & Hourglass & & $2.88^{\mathrm{a}}$ & 0.45 \\
\hline & Pear & & $2.63^{\mathrm{b}}$ & 0.43 \\
\hline \multirow{3}{*}{ Body Size } & Underweight & & $3.52^{\mathrm{a}}$ & 0.69 \\
\hline & Normal & & $2.84^{\mathrm{b}}$ & 0.53 \\
\hline & Overweight & & $1.64^{\mathrm{c}}$ & 0.51 \\
\hline
\end{tabular}

$* p<.05, * * * p<.001$

Post-hoc comparison: $\mathrm{a}>\mathrm{b}>\mathrm{c}$ 
ma: $3.7 \%, n=4$; California: $14.0 \%, n=15$ ) than the California sample group.

\section{Main Effect}

The results showed significant main effects of region, body shape, and body size on the perception of attractiveness (Table 1). The region of the participants had a significant main effect on the attractiveness perception. Participants in California rated the body scan images more attractive than participants in Alabama $(F=13.92, p<.001)$. The body shape of body scan images had a significant main effect on the attractiveness perception. Hourglass shapes were rated significantly more attractive than rectangle shapes and pear shapes $(F=72.84, p<.001)$, respectively. The perceived attractiveness levels of the rectangle shapes and the pear shapes were similar. The body size of body scan images had a significant main effect on the attractiveness perception. Underweight images were rated significantly more attractive than the normal weight images, and the normal weight images were rated significantly more attractive than the overweight images $(F=1114.02$,

Table 2. Means of attractiveness according to region and body shape

\begin{tabular}{c|c|c|c}
\hline \hline \multirow{2}{*}{ Region } & \multicolumn{3}{|c}{ Body Shape } \\
\cline { 2 - 4 } & Rectangle & Hourglass & Pear \\
\cline { 2 - 4 } & $M(S D)$ & $M(S D)$ & $M(S D)$ \\
\hline AL & $2.56(0.43)$ & $2.77(0.41)$ & $2.51(0.39)$ \\
\hline $\mathrm{CA}$ & $2.68(0.41)$ & $2.99(0.46)$ & $2.74(0.44)$ \\
\hline
\end{tabular}

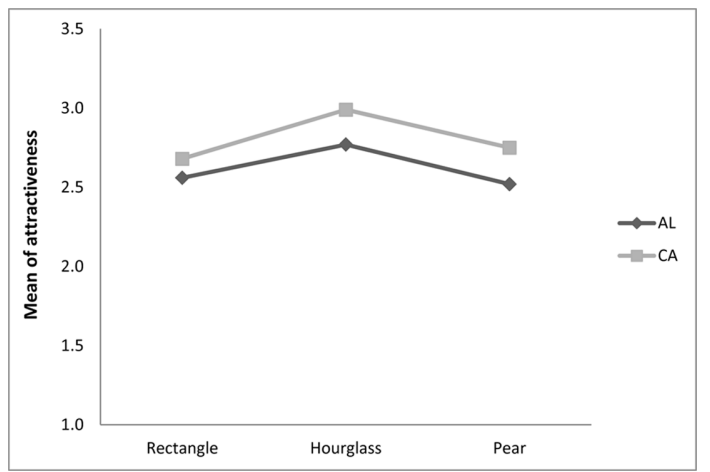

Fig. 2. Means of attractiveness according to region and body shape. $p<.001)$. This reflects the current cultural norms in society, as hourglass shape and small body size dominate how women are portrayed in mass media.

\section{Two-Way and Three-Way Interaction Effect}

A significant two-way interaction effect was found between the region of the participants and the body shape of body scan images $(F=3.09, p<.05)$ (Table 1 , Table 2, and Fig. 2). The increased attractiveness perception level in the hourglass and pear shapes compared to the rectangle shapes found for participants in California was greater than for participants in Alabama. In other words, participants in California perceived the hourglass shapes and pear shapes, compared to the rectangle shapes, far more attractive than the participants in Alabama. Additionally, participants in California perceived the rectangle shapes less attractive than the pear shapes, but participants in Alabama perceived the pear shapes less attractive than the rectangle shapes. However, participants in both regions perceived the hourglass shapes the most attractive among all three shapes.

No significant two-way interaction effect was found between the region of the participants and the body

Table 3. Means of attractiveness according to region and body size

\begin{tabular}{c|c|c|c}
\hline \hline \multirow{2}{*}{ Region } & \multicolumn{3}{|c}{ Body Size } \\
\cline { 2 - 4 } & Underweight & Normal & Overweight \\
\cline { 2 - 4 } & $M(S D)$ & $M(S D)$ & $M(S D)$ \\
\hline $\mathrm{AL}$ & $3.58(0.53)$ & $2.73(0.50)$ & $1.54(0.43)$ \\
\hline $\mathrm{CA}$ & $3.72(0.53)$ & $2.96(0.53)$ & $1.74(0.56)$ \\
\hline
\end{tabular}

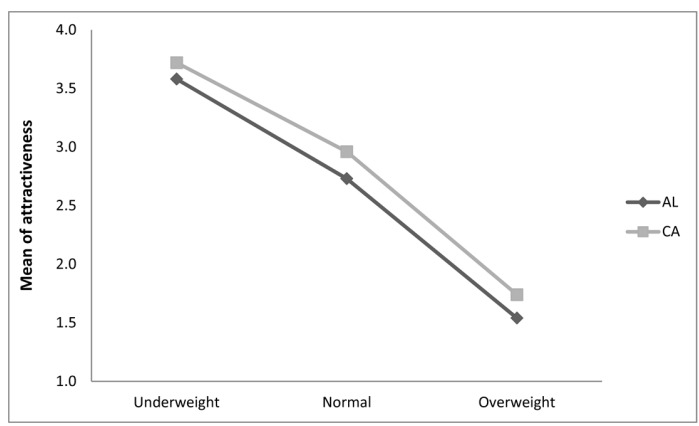

Fig. 3. Means of attractiveness according to region and body size. 
size of body scan images $(F=0.46)$ (Table 1 , Table 3 , and Fig. 3). The results indicate that both California and Alabama participants perceived underweight size images more attractive than the normal weight images and the normal weight images more attractive than the overweight images.

A significant two-way interaction effect was found between body size and body shape $(F=90.28, p<.001)$ (Table 1, Table 4, and Fig. 4). For overweight images, pear shapes were perceived more attractive than the rectangle and hourglass shapes. For underweight and normal weight images, the hourglass shapes were perceived more attractive than the rectangle and pear shapes. It is possible that the pear shapes in overweight ranges looked smaller in size than the hourglass and rectangle shapes in overweight ranges because a pear shape has a smaller upper body than a lower body; since participants generally perceived smaller body size images more attractive, they could have perceived pear shapes in overweight category more attractive than the other shapes in overweight category. However, regardless of the body shapes, participants perceived underweight images more attractive than the normal weight images, and the normal weight images more attrac-

Table 4. Means of attractiveness according to body size and body shape

\begin{tabular}{c|c|c|c}
\hline \hline \multirow{2}{*}{ Body Size } & \multicolumn{3}{|c}{ Body Shape } \\
\cline { 2 - 4 } & Rectangle & Hourglass & Pear \\
\cline { 2 - 4 } & $M(S D)$ & $M(S D)$ & $M(S D)$ \\
\hline Underweight & $3.52(0.69)$ & $3.89(0.66)$ & $3.54(0.58)$ \\
\hline Normal & $2.73(0.61)$ & $3.24(0.71)$ & $2.56(0.55)$ \\
\hline Overweight & $1.61(0.53)$ & $1.52(0.61)$ & $1.78(0.58)$ \\
\hline
\end{tabular}

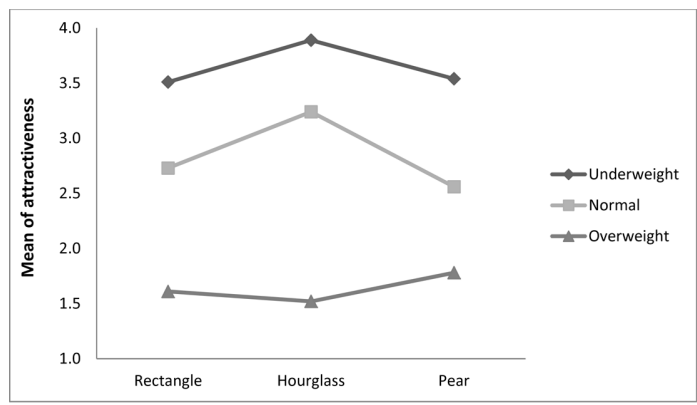

Fig. 4. Means of attractiveness according to body size and body shape. tive than the overweight images. No significant threeway interaction effect was found between region, body shape and body size.

\section{Conclusions and Implications}

The current study successfully examined the influence of body size, body shape, and region on the perception of attractiveness. Previous research on female attractiveness largely used 2D line drawings or pictures as stimuli and used waist and hip as a measure of female body shape. More recent studies have used different types of stimuli such as 3D videos (Rilling et al., 2009), digitally manipulated images (Gründl et al., 2009; Kościńnki, 2013; Smith et al., 2007b), and 3D body scanned images (Brooks et al., 2010; Price et al., 2013). Our study also took a recent approach of creating stimuli with a 3D body scanner and examined overall body shape determined by shoulder, waist, and hip variables.

This study shows that both body shape and body size influence people's perception of attractiveness, supporting both directions of the literature - Singh (2006) a proponent of body shape, and Swami et al. (2005, 2007), proponents of body size. However, body size played as a more important factor than did the body shape when participants determined the attractiveness level of female figures. These results coincides with previous studies by Kościński (2013) and Smith et al. (2007b); they found that both BMI and WHR influenced the perception of female attractiveness but concluded BMI to be a more important factor than WHR when perceiving female attractiveness. In other words, although hourglass shapes were perceived to be the most attractive shape and underweight sizes were perceived to be the most attractive size in this study, the hourglass shape will lose its significance once body size gets larger and people will probably perceive hourglass shape to be the most attractive body shape only when body size is small.

The study findings also indicate that some regional differences exist between the two sample groups with regards to their perceptions of attractiveness. Overall, California participants rated the body scan images more attractive than Alabama participants. This may 
be attributed to the fact that the California sample group in this study had a higher rate of ethnic diversity than Alabama and it is plausible to suggest California's culture contributed toward a higher tolerance in that group's perceptions of attractiveness regarding various body shapes and body sizes in general. This finding sup-ports Fallon (1990)'s belief about attractiveness that each cultural group has different beauty standards and the perception of attractiveness varies from culture to culture. Also, the result is in a same line with Swami et al. (2010)'s cross-cultural study in a way that cross-cultural differences exist in evaluation of ideal female figure.

The present study suggests that researchers should focus on body shape from a larger viewpoint rather than treating it as simple WHR. In addition, representation of more realistic images of real women in a wider range of BMI and body shape will maximize the validity of findings and provide stronger understanding of the role of each in perceptions of attractiveness. Although images of real people obtained from a body scanner were used in this study, they were presented on a screen, which restricted the full impression of a 3D view. For further research in this field, use of body scan images that can be fully rotatable in 360 degree is recommended.

The participants of the study were young female college students; these young women's view on what makes ideal and attractive women was in agreement with what is depicted as the current culture's ideal in the mass media (Swami et al., 2010). Such perception of ideal figure is closely related to people's appearance-management behavior such as cosmetic use, diet, plastic surgery, and apparel selection (Rudd \& Lennon, 2000; Tiggemann \& Andrew, 2012). Studies found out that women use clothes to attain a slender hourglass ideal look (Grogan et al., 2013; Rudd \& Lennon, 2000). Alongside with the intervention on more realistic and healthier body ideals (Swami et al., 2010), promoting clothes as a mean of more positive appearance-management behavior can be encouraged rather than leaving women practicing a risky appearance-management behavior such as an extreme diet or plastic surgery. Such studies of perception of female attractiveness related to apparel selection will be a valuable to- pic of continuing future study for researchers in both Textiles and Apparel field and Psychology field.

\section{References}

Aghekyan, M., Ulrich, P., \& Connell, L. J. (2012). Using body scans in assessing perceptions of body attractiveness and size: cross-cultural study. International Journal of Fashion Design, Technology and Education, 5(2), 81-92.

Berg, F. M. (2000). Women afraid to eat: Breaking free in today's weight-obsessed world. Hettinger, SA: Health Weight Network.

Brooks, R., Shelly, J. P., Fan, J., Zhai, L., \& Chau, D. K. P. (2010). Much more than a ratio: Multivariate selection on female bodies. Journal of Evolutionary Biology, 23 (10), 2238-2248.

Bush, H., Williams, R., Lean, M., \& Anderson, A. (2001). Body image and weight consciousness among South Asian, Italian and general population women in Britain. Appetite, 37(3), 207-215.

Connell, L. J., Ulrich, P. V., Brannon, E. L., Alexander, M., \& Presley, A. B. (2006). Body shape assessment scale: Instrument development for analyzing female figures. Clothing and Textiles Research Journal, 24(2), 80-95.

Dixson, B. J., Dixson, A. F., \& Baoguo, L. I. (2010a). Female waist-to-hip ratio, body mass index and sexual attractiveness in China. Current Zoology, 56(2), 1-17.

Dixson, B. J., Dixson, A. F., Bishop, P. J., \& Parish, A. (2010b). Human physique and sexual attractiveness in men and women: A New Zealand-US comparative study. Archives of Sexual Behavior, 39(3), 798-806.

Dixson, B. J., Grimshaw, G. M., Linklater, W. L., \& Dixson, A. F. (2010c). Watching the hourglass eye tracking reveals men's appreciation of the female form. Human Nature-an Interdisciplinary Biosocial Perspective, 21(4), 355-370.

Fallon, A. (1990) Culture in the mirrors: Sociocultural determinants of body image. In T. F. Cash \& T. Pruzinsky (Eds.), Body images: Development, deviance, and change (pp. 81-107). New York: Guilford Press.

Forestell, C. A., Humphrey T. M., \& Stewart, S. H. (2004). Involvement of body weight and shape factors in rating of attractiveness by women: A replication and extension of Tassinary and Hansen (1998). Personality and Individual Differences, 36(2), 295-305.

Furnham, A., \& Alibhai, N. (1983). Cross-cultural differences in the perception of female body shapes. Psychological Medicine, 13(4), 829-837.

Furnham, A., \& Baguma, P. (1994). Cross-cultural differences in the evaluation of male and female body shapes. International Journal of Eating Disorders, 15, 81-89.

Furnham, A., \& Nordling, R. (1998). Cross-cultural differences in preferences for specific male and female body shapes. Personality and Individual Differences, 25(4), 635- 
648.

Furnham, A., Tan, T., \& McManus, C. (1997). Waist-to-hip ratio and preferences for body shape. A replication and extension. Personality Individual Differences, 22(4), 539549.

Grogan, S., Gill, S., Brownbridge, K., Kilgariff, S., \& Whalley, A. (2013). Dress fit and body image: A thematic analysis of women's accounts during and after trying on dresses. Body Image, 10(3), 380-388.

Gründl, M., Eisenmann-Klein, M., \& Prantl, L. (2009). Quantifying female bodily attractiveness by a statistical analysis of body measurements. Plastic and Reconstructive Surgery, 123(3), 1064-1071.

Gupta, M., Chaturvedi, S., Chandarana, P., \& Johnson, A. (2001). Weight-related body image concerns among 1824-year-old women in Canada and India: An empirical comparative study. Journal of Psychosomatic Research, 50(4), 193-198.

Henss, R. (1995). Waist-to-hip ratio and attractiveness. A replication and extension. Personality and Individual Differences, 19(4), 479-488.

Hogg, M. A., \& Graham, M. V. (1995). Social physiology: An introduction. London: Prentice Hall, Harvester Wheatsheaf.

Jung, J., \& Lennon, J. S. (2003). Body image, appearance self-schema, and media images. Family and Consumer Sciences Research Journal, 32(1), 27-51.

Jung, J., \& Peterson, M. (2007). Body dissatisfaction and patterns of media use among preadolescent children. $\mathrm{Fa}$ mily and Consumer Sciences Research Journal, 36(1), 40-54.

Kościński, K. (2013). Attractiveness of womens body: Body mass index, waisthip ratio, and their relative importance. Behavioral Ecology, 24(4), 914-925.

Mazur, A. (1986). U.S. trends in feminine beauty and overadaptation. Journal of Sex Research, 22(3), 281-303.

Price, M. E., Pound, N., Dunn, J., Hopkins, S., \& Kang, J. (2013). Body shape preferences: Associations with rater body shape and sociosexuality. Plos One, 8(1), e52532.

Rilling, J. K., Kaufman, T. L., Smith, E. O., Patel, R., \& Worthman, C. M. (2009). Abdominal depth and waist circumference as influential determinants of human female attractiveness. Evolution and Human Behavior, 30(1), 21-31.

Rudd, N. A., \& Lennon, S. J. (2000). Body image and appearance-management behaviors in college women. Clothing and Textiles Research Journal, 18(3), 152-162.

Singh, D. (1993). Adaptive significance of female physical attractiveness: Role of waist-to-hip ratio. Journal of Personality and Social Physiology, 65(2), 293-307.

Singh, D. (1994a). Waist-to-hip ratio and judgment of attractiveness and healthiness of female figures by male and female physicians. International Journal of Obesity, 18 (11), 731-737.
Singh, D. (1994b). Is thin really beautiful and good? Relationship between waist-to-hip ratio (WHR) and female attractiveness. Personality and Individual Differences, $16,123-192$.

Singh, D. (2006). Universal allure of the hourglass figure: An evolutionary theory of female physical attractiveness. $\mathrm{Cli}$ nics in Plastic Surgery, 33, 359-370.

Singh, D., \& Singh, D. (2011). Shape and significance of feminine beauty: An evolutionary perspective. Sex Roles, 64(9-10), 723-731.

Smith, K. L., Cornelissen, P. L., \& Tovée, M. J. (2007a). Color 3D bodies and judgments of human female attractiveness. Evolution and Human Behavior, 28(1), 48-54.

Smith, K. L., Tovee, M. J., Hancock, P. J. B., Bateson, M., Cox, M. A. A., \& Cornelissen, P. L. (2007b). An analysis of body shape attractiveness based on image statistics: Evidence for a dissociation between expressions of preference and shape discrimination. Visual Cognition, 15 (8), 927-953.

Swami, V., \& Tovée, M. J. (2005). Male physical attractiveness in Britain and Malaysia: A cross-cultural study. Body Image, 2(4), 383-393.

Swami, V., \& Tovée, M. J. (2007). The relative contribution of profile body shape and weight to judgments of women's physical attractiveness in Britain and Malaysia. Body Image, 4(4), 391-396.

Swami, V., Frederick, D. A., Aavik, T., Alcalay, L., Allik, J., Anderson, D., ... Zivcic-Becirevic, I. (2010). The attractive female body weight and female body dissatisfaction in 26 countries across 10 world regions: Results of the international body project I. Personality and Social Psychology Bulletin, 36(3), 309-325.

Tassinary, L. G., \& Hansen, K. A. (1998). A critical test of the waist-to-hip ratio hypothesis of female physical attractiveness. Physiological Science, 9(2), 150-155.

Tiggemann, M., \& Andrew, R. (2012). Clothing choices, weight, and trait self-objectification. Body Image, 9(3), 409412.

Toveé, M. J., Benson, P. J., Emery, J. L., Mason, S. M., \& Cohen-Toveé, E. M. (2003). Measurement of body size and shape perception in eating-disordered and control observers using body-shape software. British Journal of Physiology, 94(4), 501-516.

Toveé, M. J., Hancock, P. J. B., Mahmoodi, S., Singleton, B. R., \& Cornelissen, P. L. (2002). Human female attractiveness: Waveform analysis of body shape. Proceeding of the Royal Society of London B, 269(1506), 2205-2213.

Toveé, M. J., Maisey, S., Emery, J. L., \& Cornelissen, P. L. (1999). Visual cues to female physical attractiveness. Proceedings of the Royal Society of London B, 266(1415), 211-218.

Toveé, M. J, Reinhardt, S., Emery, J. L., \& Cornelissen, P. L. (1998). Optimal BMI and maximal sexual attractiveness. The Lancet, 352(9127), 548. 
United States Census Bureau. (n.d.). State \& County QuickFacts. United States Census Bureau. Retrieved July 25, 2013 from http://quickfacts.census.gov/qfd/index.html

Wilson, J. M. B., Tripp, D. A., \& Boland, F. J. (2005). The relative contributions of subjective and objective mea- sures of body shape and size to body image and disordered eating in women. Body Image, 2(3), 233-247.

World Health Organization. (n.d.). BMI Classification. World Health Organization. Retrieved July 25, 2013 from http:// apps.who.int/bmi/index. jsp?introPage=intro_3.html 